\title{
PERTURBATION THEORY FOR GENERALIZED FREDHOLM OPERATORS. II
}

\author{
S. R. CARADUS ${ }^{1}$
}

\begin{abstract}
If $T$ is a bounded linear operator with generalized inverse $S$, i.e. $T S T=T$, we obtain conditions so that $T-U$ has a generalized inverse. When $T$ is a Fredholm operator, the conditions become simply the requirement that $I-U S$ (or equivalently $I-S U$ ) is a Fredholm operator. This result includes the classical perturbation theorems where $U$ is required to have a small norm or to be compact.
\end{abstract}

Let $X$ and $Y$ denote Banach spaces and $B(X, Y)$, the continuous linear operators defined on $X$ with range in $Y$. We will write $G F(X, Y)$ for the set of (generalized Fredholm) operators $T$ in $B(X, Y)$ such that $N(T)$, the nullspace and $R(T)$, the range, are closed complemented subspaces of $X$ and $Y$, respectively. It is well known that $T \in G F(X, Y)$ if and only if there exists $S \in B(Y, X)$ such that $T S T=T$. Such an $S$ is called a generalized inverse of $T$ and it is an easy exercise to show that $T S$ is a projection of $Y$ onto $R(T)$ and $I-S T$ is a projection of $X$ onto $N(T)$. Hence Fredholm operators can be characterized as those $T$ in $G F(X, Y)$ such that $I-T S$ and $I-S T$ are finite rank projections whenever $S$ is a generalized inverse of $T$. (In our earlier paper [2], considerations were limited to the case $X=Y$ and a generalized inverse was required to satisfy the additional conditions $S T S=S$. However, it is easy to verify that if $T S T=T$ then the operator $S^{\prime}=S T S$ satisfies both conditions so that $G F(X, Y)$ is not changed by the additional condition. For further information on this subject, see [3].)

The problem studied in [2] was: if $T \in G F(X)$, when does $T-U$ $\in G F(X)$ ? Conditions of a rather general kind were obtained, which included earlier results due to Dieudonné, Crownover and Saphar as special cases. However, our results did not include the standard perturbation theorems of Fredholm theory. We now proceed to develop the theory sufficiently far to subsume and unify this classical material. It will also become clear that the generalized inverse plays a natural role in perturbation theory.

We first need a Lemma of Atkinson [1] whose simple proof is given because it provides information needed later.

Lemma 1. If $C$ and $V$ belong to $B(X)$ and $C V C-C$ belongs to $G F(X)$ then $C$ belongs to $G F(X)$.

Received by the editors October 24, 1975.

AMS (MOS) subject classifications (1970). Primary 47B30.

${ }^{1}$ Research partly supported by NRC Operating Grant A3985. 
Proof. Suppose $(C V C-C) S(C V C-C)=C V C-C$. Then, multiplying out and rearranging, we get $C(V-V C S C V+S C V+V C S-S) C=C$.

REMARK (1). Suppose $C V C-C$ is an operator $F$ of finite rank. Then

$$
\begin{aligned}
C(V-V C S C V & +S C V+V C S-S) \\
& =C V-(C+F) S C V+C S C V+(C+F) S-C S \\
& =C V+\text { operator of finite rank. }
\end{aligned}
$$

Similarly

$$
(V-V C S C V+S C V+V C S-S) C=V C+\text { operator of finite rank. }
$$

Hence the projections onto $R(C)$ and $N(C)$, respectively, differ from $C V$ and $I-V C$ by operators of finite rank.

Lemma 2. Suppose $U \in B(X, Y)$ and $V \in B(Y, X)$. Then $I-U V$ is Fredholm if and only if $I-V U$ is Fredholm.

Proof. Suppose $I-U V$ is Fredholm. Then there exists $S \in B(Y)$ such that

$$
(I-U V) S(I-U V)=I-U V
$$

and $I-S(I-U V), I-(I-U V) S$ are both finite rank operators. Now (1) can be multiplied out to give

$$
S-U V S-S U V+U V S U V=I-U V .
$$

Moreover, as Atkinson observed in [1], we have

$$
\begin{aligned}
(I-V U) & (I+V S U)(I-V U) \\
& =I-V[I-(S-U V S-S U V+U V S U V-I+U V)] U \\
& =I-V U \text { using (2). }
\end{aligned}
$$

Hence, $I+V S U$ is a generalized inverse of $I-V U$, and from

$$
\begin{aligned}
& I-(I-V U)(I+V S U)=V[I-(I-U V) S] U \text { and } \\
& I-(I+V S U)(I-V U)=V[I-S(I-U V)] U,
\end{aligned}
$$

we see that the associated projections are finite dimensional. Hence $I-V U$ is Fredholm.

Note. For the case $X=Y$, this lemma is contained in Theorem 1 of [1].

THEOREM. Let $T$ be an operator in $G F(X, Y)$ with generalized inverse $S$. Suppose that $U \in B(X, Y)$ and that $I-U S$ is a Fredholm operator with generalized inverse $V$. Finally, suppose that $(I-T S) V U(I-S T)$ belongs to $G F(X, Y)$. Then $T-U$ belongs to $G F(X, Y)$. 
Proof. Since $I-U S$ is Fredholm, the operators

$$
F_{1}=I-(I-U S) V \text { and } F_{2}=I-V(I-U S)
$$

are finite dimensional projections. Then we have

$$
\begin{aligned}
T-U & -(T-U) S V(T-U) \\
& =[I-U S-(T-U) S] V(T-U)+F_{1}(T-U) \\
& =(I-T S) V[(I-U S) T-U(I-S T)]+F_{1}(T-U) \\
& =(I-T S)[V(I-U S) T-V U(I-S T)]+F_{1}(T-U) \\
& =(I-T S)\left[\left(I-F_{2}\right) T-V U(I-S T)\right]+F_{1}(T-U) \\
& =(I-T S) T-(I-T S) V U(I-S T)-(I-T S) F_{2} T+F_{1}(T-U) \\
& =-(I-T S) V U(I-S T)+\text { operator of finite rank. }
\end{aligned}
$$

The conclusion now follows from Lemma 1 .

REMARKS (2). By Lemma 2, the assumption " $I-U S$ is Fredholm" could be replaced by " $I-S U$ is Fredholm". If this is the case and $W$ is a generalized inverse of $I-S U$, then $T-U-(T-U) W S(T-U)$ reduces to $-(I-T S) U W(I-S T)$ plus an operator of finite rank.

(3) In [2], we assumed that $\|U\|<\|S\|^{-1}$. In this case, $I-U S$ and $I-S U$ are both invertible.

(4) Suppose now that $T$ is generalized Fredholm and $R(T)$ has finite codimension. Then $I-T S$ is a finite rank projection when $S$ is a generalized inverse of $T$. Hence $(I-T S) V U(I-S T)$ is an operator of finite rank and therefore in $G F(X, Y)$. Therefore from Theorem 1, we can conclude that if $I-U S$ is a Fredholm operator then $T-U$ belongs to $G F(X, Y)$. We can also show that $R(T-U)$ has finite codimension: we know that $T-U-$ $(T-U) S V(T-U)$ has finite rank, hence by Remark (1), the projection $P$ onto $R(T-U)$ differs from $(T-U) S V$ by a finite rank operator. Hence

$$
\begin{aligned}
I-P & =I-(T-U) S V+\text { finite rank operator } F \\
& =I-(T S-I+I-U S) V+F=I-(T S-I) V-\left(I-F_{1}\right)+F \\
& =(I-T S) V+F_{1}+F
\end{aligned}
$$

which has finite rank. Similarly, if $T$ is generalized Fredholm and $N(T)$ has finite dimension, we can derive a similar conclusion, using the equivalent assumption that $I-S U$ is Fredholm. Hence we obtain the important extension of Fredholm theory:

COROLlARY. Suppose $T$ is a generalized Fredholm operator with generalized inverse $S$. Let I-US be a Fredholm operator. Then if $R(T)$ has finite codimension it follows that $T-U$ is a generalized Fredholm operator with $R(T-U)$ having finite codimension; if $N(T)$ has finite dimension, it follows that 
$T-U$ is a generalized Fredholm operator with $N(T-U)$ having finite dimension.

Remarks (5). We observe that the condition " $I-U S$ is Fredholm" subsumes the usual two conditions of the classical theory. For if $\|U\|$ is small, we get $I-U S$ invertible, while if $U$ is compact, we get $U S$ compact and hence $I-U S$ Fredholm. Also if $U$ belongs to any ideal which consists of Riesz operators, then $I-U S$ will be Fredholm. The largest ideal of Riesz operators is the so-called "inessential" operators; therefore we have obtained another known perturbation result: if $T \in G F(X)$ and $N(T)$ is finite dimensional (resp. $R(T)$ has finite codimension), and $U$ is an inessential operator, then $T-U \in G F(X)$ and $N(T-U)$ is finite dimensional (resp. $R(T-U$ ) has finite codimension). (See [4, pp. 98-102] for further information on this topic.)

(6) When $X=Y$, we can obtain the above results much more easily. Let $K$ denote the ideal of compact operators in $B(X)$ and $\pi: B(X) \rightarrow B(X) / K$ the canonical homomorphism. Then if $\mathcal{G}_{l}$ denotes the set of left invertible elements in $B(X) / K$, it is well known $\left[4\right.$, p. 66] that $\pi^{-1}\left(\mathcal{G}_{l}\right)$ is precisely the set of operators $T$ in $G F(X)$ with $N(T)$ finite dimensional. Moreover the Fredholm operators are given by $\pi^{-1}(\mathcal{G})$ where $\mathcal{G}$ is the set of invertible operators in $B(X) / K$. Hence, if $T \in G F(X)$ with generalized inverse $S$ and $N(T)$ finite dimensional, if $U \in B(X)$ with $I-S U$ Fredholm, we have $\pi(S) \pi(T)=\pi(I)$ and hence $\pi(S)[\pi(T)-\pi(U)]=\pi(I-S U)$. Hence $\pi(T-U)$ is left invertible and so $T-U \in G F(X)$ with $N(T-U)$ finite dimensional. When $X \neq Y$, such arguments cannot be extended.

But clearly the new condition allows many other types of perturbation. For example, if $N(U)+N(T)=X$, then we can find a subspace $X_{1}$ such that $X_{1} \oplus N(T)=X$ and $N(U) \supseteq X_{1}$. Then if $P$ is any projection of $Y$ onto $R(T)$ and $T_{1}$ denotes the restriction of $T$ to $X_{1}$, then $S=T_{1}^{-1} P$ is a generalized inverse of $T$. Now $R(S)=X_{1}$ so that $U S=0$. Hence $I-U S$ is certainly Fredholm. Similarly, if $R(U)+R(T)=Y$, we can construct $S$ such that $N(S) \supseteq R(U)$ so that again $I-S U$ is Fredholm.

Another way to insure that $I-U S$ is Fredholm: assume that $U$ and $S$ "almost commute" i.e. $U S-S U$ is compact, and that the Fredholm spectrum $\{\lambda \in C: \lambda I-U$ is not Fredholm $\}$ is contained in a small disc with centre $\lambda=0$. Then $\pi(I-U S)=\pi(I)-\pi(U) \pi(S)$ is invertible because $\pi(U)$ and $\pi(S)$ commute and $\pi(U)$ has small spectrum. Thus $\pi(U) \pi(S)$ also has small spectrum excluding the point $\lambda=1$.

(7) The above method obtains results for operators $T$ with $N(T)$ finite dimensional and $R(T)$ closed and complemented, or $R(T)$ with finite codimension and $N(T)$ complemented. However, the classical theory deals also with the somewhat larger class where the complementation condition is omitted i.e. the semi-Fredholm operators. In Hilbert space the two classes coincide, of course, but in general, our methods cannot be extended.

(8) The algebraic nature of the arguments developed above suggest that the most natural setting for the study of generalized Fredholm operators is a suitable type of locally convex vector space. In particular, the theory of saturated operator algebras as developed in the recent monograph [5] of $\mathbf{H}$. Heuser allows for a natural generalization. 


\section{REFERENCES}

1. F. V. Atkinson, On relatively regular operators, Acta. Sci. Math. (Szeged) 15 (1953), 38-56. MR 15, 134.

2. S. R. Caradus, Perturbation theory for generalized Fredholm operators, Pacific J. Math. 52 (1974), 11-15. MR 50 \# 5520.

3. - Operator theory of the pseudo-inverse, Queen's Papers in Pure and Appl. Math., no. 38, Queen's University, Kingston, Ontario.

4. S. R. Caradus, W. E. Pfaffenberger and B. Yood, Calkin algebras and algebras of operators on Banach spaces, Dekker, New York, 1974.

5. H. Heuser, Funktional Analysis, Stuttgart, 1975.

Department of Mathematics, QueEn's University, Kingston, Ontario, Canada 